\title{
Volumetric change of the latissimus dorsi muscle after immediate breast reconstruction with an extended latissimus dorsi musculocutaneous flap
}

\author{
Su Bong Nam ${ }^{1}$, Heung Chan $\mathrm{Oh}^{1}$, Jae Yeon $\mathrm{Choi}^{1}$, Seong Hwan Bae ${ }^{1}$, Ki Seok $\mathrm{Choo}^{2}$, \\ Hyun Yul Kim³, Sang Hyup Lee ${ }^{3}$, Jae Woo Lee ${ }^{1}$ \\ Departments of ${ }^{1}$ Plastic and Reconstructive Surgery, ${ }^{2}$ Radiology, and ${ }^{3}$ Surgery, Pusan National University School of Medicine, Yangsan, Korea
}

Background In immediate breast reconstruction using an extended latissimus dorsi musculocutaneous (eLDMC) flap, the volume of the flap decreases, which causes a secondary deformity of the breast shape. Since little research has investigated this decrease in muscle volume, the authors conducted an objective study to characterize the decrease in muscle volume after breast reconstruction using an eLDMC flap.

Methods Research was conducted from October 2011 to November 2016. The subjects included 23 patients who underwent mastectomy due to breast cancer, received immediate reconstruction using an eLDMC flap without any adjuvant chemotherapy or radiotherapy, and received a computed tomography (CT) scan from days 7 to 10 after surgery and 6 to 8 months postoperatively. In 10 patients, an additional CT scan was conducted 18 months postoperatively. Axial CT scans were utilized to measure the volumetric change of the latissimus dorsi muscle during the follow-up period.

Results In the 23 patients, an average decrease of 54.5\% was observed in the latissimus dorsi muscle volume between the images obtained immediately postoperatively and the scans obtained 6 to 8 months after surgery. Ten patients showed an average additional decrease of $11.9 \%$ from 6-8 months to 18 months after surgery.

Conclusions We studied changes in the volume of the latissimus dorsi muscle after surgery using an eLDMC flap performed after a mastectomy without adjuvant chemotherapy or radiotherapy. In this study, we found that immediate breast reconstruction using a latissimus dorsi muscle flap led to a decrease in muscle volume of up to $50 \%$.

Keywords Mammaplasty / Superficial back muscles / Myocutaneous flap
Correspondence: Jae Woo Lee Department of Plastic and Reconstructive Surgery, Pusan National University School of Medicine, 49 Busandaehak-ro, Mulgeum-eup, Yangsan 50612, Korea Tel: $+82-55-360-2573$

Fax: +82-55-360-2158

E-mail: surgeon@pusan.ac.kr

This work was supported by a 2015 clinical research grant from Pusan National University Yangsan Hospital.

Received: 15 Nov 2017 Revised: 20 Sep $2018 \bullet$ Accepted: 22 Oct 2018

pISSN: 2234-6163 • elSSN: 2234-6171 • https://doi.org/10.5999/aps.2017.01690 • Arch Plast Surg 2019;46:135-139

\section{INTRODUCTION}

Studies on the early detection and appropriate treatment of breast cancer have rapidly progressed in recent years, and active research in this field has led to the gradual recognition that breast reconstruction after mastectomy should be considered mandatory, instead of merely an option available to breast surgeons. Delayed reconstruction was generally conducted in the past, but immediate reconstruction is known to be a better method when patient satisfaction and cosmetic outcomes are 
considered [1-3].

Immediate breast reconstruction is most commonly performed using the latissimus dorsi musculocutaneous (LDMC) flap and transverse rectus abdominis musculocutaneous (TRAM) flap. The TRAM flap is beneficial because it provides excellent results in reconstruction procedures of large breasts, without requiring implants to be used. However, the TRAM flap has the drawbacks of causing greater donor site morbidity than the LDMC flap and requiring a longer operative time $[2,3]$. Satisfactory results using only the LDMC flap can be expected for reconstructions of small breasts. However, using implants with the LDMC flap for moderately-sized to large breasts has been reported to lead to better results than reconstruction with an extended LDMC flap for reasons relating to donor site morbidity [4-6].

When conducting immediate breast reconstruction using an LDMC flap, the motor nerve is ligated to avoid synkinetic muscle contracture. However, this procedure can cause latissimus dorsi muscle atrophy resulting from denervation, and it can also make it difficult to select the appropriate implant size for breast reconstructions in which implants are needed [7-9]. The primary objective of breast reconstruction is to achieve symmetry with the other breast. In order to accomplish this goal, the extent of pure muscle atrophy resulting from denervation must be accurately understood. The authors conducted an objective study among patients who did not receive adjuvant therapy, such as chemotherapy or radiation therapy, and analyzed postoperative volumetric changes of the latissimus dorsi muscle through computed tomography (CT) scans.

\section{METHODS}

Research was conducted from October 2011 to November 2016 with Institutional Review Board approval (IRB No. 052018-051). Twenty-three patients were selected who received total mastectomy at the Pusan National University Yangsan Hospital and then underwent immediate breast reconstruction using an LDMC flap performed by a single plastic surgeon. All subjects received follow-up CT scans using the same protocol, and provided written informed consent for the CT scans and photography ( $120 \mathrm{kVp}, 5.0 \mathrm{~mm}$ section thickness) (Discovery CT750 HD; GE Medical Systems, Milwaukee, WI, USA).

After mastectomy and axillary dissection were performed, the thoracodorsal pedicle pathway was confirmed through the axilla and the volume and range of the removed breast tissue were checked. A skin paddle was designed with a width of 3 to $5 \mathrm{~cm}$ and a length of 10 to $20 \mathrm{~cm}$. For breast reconstructions in moderately-sized to large breasts, various types of implants were used simultaneously (Table 1). The dissection was performed using a standard method. After complete division of the muscle near its origin, a thoracodorsal pedicle was completely released and isolated. The motor nerve that inserts through the latissimus dorsi muscle was confirmed to be present in the pedicle, and it was ligated at the proximal part and excised to be $1 \mathrm{~cm}$ in length in order to prevent synkinetic contracture of the muscle after the operation. After shifting the patient to the supine position, the upper body was elevated $60^{\circ}$ to a near-sitting position. The latissimus dorsi muscle was folded and secured, considering the width and volume of the defect. When a portion of the skin of the breast or the nipple-areolar complex (NAC) was removed, the skin paddle was positioned outwardly. Even when the NAC was preserved, part of the de-epithelialized skin paddle was always positioned inferior to the NAC in order to maintain NAC projection after surgery.

CT scans were conducted with the consent of all patients 7 to 10 days after surgery, and again 6 to 8 months after surgery. Ten patients required additional CT scans 18 months after surgery. The muscle volume was measured using the axial view in a picture archiving and communications system (PACS). Each image showed the transpositioned latissimus dorsi muscle, and the cross-section of the muscle was manually measured using the built-in measurement tool in the PACS. These values were multiplied by $5 \mathrm{~mm}$ of thickness and added together to obtain the volume (Fig. 1). Two plastic surgeons (SBN and JWL) and one radiologist (KSC) conducted the measurements of the area and volume of the cross-sections, and the average values were calculated and used as representative values.

\section{RESULTS}

In the 23 patients, the initial latissimus dorsi muscle volume that was measured 7 to 10 days after surgery ranged from $64.24 \mathrm{~cm}^{3}$ to $177.31 \mathrm{~cm}^{3}$, with an average of $118.82 \mathrm{~cm}^{3}$. The volume measured 6 to 8 months after surgery ranged from $25.5 \mathrm{~cm}^{3}$ to $107.19 \mathrm{~cm}^{3}$, with an average of $54.09 \mathrm{~cm}^{3}$. The extent to which the volume decreased varied considerably across cases, from $32.4 \%$ to $68.7 \%$. The average volume decrease by 6 to 8 months after surgery was $54.5 \%$. In the 10 patients who underwent additional CT scans 18 months after surgery, we found an additional volume decrease of $1.3 \%$ to $27.7 \%$ (average, $11.9 \%$ ) compared to their measurements from 10 to 12 months before (Table 1). Ultimately, the final decrease in volume was $58 \%$ compared to the initial latissimus dorsi muscle volume.

In terms of the postoperative clinical results at the final followup, one patient complained of depression in the upper part of the breast, and one patient was not satisfied with the results be- 
Table 1. Summary of cases

\begin{tabular}{|c|c|c|c|c|c|c|c|c|c|c|}
\hline Case no. & $\begin{array}{l}\text { Age } \\
\text { (yr) }\end{array}$ & $\begin{array}{c}\text { BMI } \\
\left(\mathrm{kg} / \mathrm{m}^{2}\right)\end{array}$ & $\begin{array}{l}\text { Implant type } \\
\text { and size }(\mathrm{mL})\end{array}$ & $\begin{array}{l}\text { Immediate } \\
\text { postoperative } \\
\text { volume }\left(\mathrm{cm}^{3}\right)\end{array}$ & $\begin{array}{c}\text { After 6-8 } \\
\text { months } \\
\left(\mathrm{cm}^{3}\right)\end{array}$ & $\begin{array}{l}\text { After } 18 \\
\text { months } \\
\left(\mathrm{cm}^{3}\right)\end{array}$ & $\begin{array}{c}\text { Volume } \\
\text { decrease } \\
(\%)^{\text {a) }}\end{array}$ & $\begin{array}{c}\text { Volume } \\
\text { decrease } \\
(\%)^{\text {b) }}\end{array}$ & $\begin{array}{l}\text { Total follow- } \\
\text { up period } \\
\text { (mon) }\end{array}$ & $\begin{array}{l}\text { Clinical } \\
\text { result }^{c}\end{array}$ \\
\hline 1 & 40 & 21.60 & - & 126.51 & 56.91 & 43.64 & 55.02 & 23.32 & 48 & Fair \\
\hline 2 & 55 & 20.76 & - & 110.88 & 40.40 & 29.91 & 63.56 & 25.97 & 60 & Good \\
\hline 3 & 43 & 18.73 & - & 64.24 & 25.50 & - & 60.31 & - & 42 & Good \\
\hline 4 & 51 & 25.48 & Saline bag, 120 & 98.39 & 38.76 & - & 60.61 & - & 37 & Good \\
\hline 5 & 40 & 30.86 & - & 158.68 & 107.19 & 95.49 & 32.45 & 10.92 & 24 & Good \\
\hline 6 & 50 & 21.64 & Round, 90 & 102.56 & 44.48 & 32.14 & 56.63 & 27.74 & 25 & Good \\
\hline 7 & 34 & 20.08 & Round, 90 & 116.03 & 36.32 & - & 68.70 & - & 29 & Good \\
\hline 8 & 35 & 21.88 & Round, 90 & 102.58 & 44.07 & 37.28 & 57.04 & 15.41 & 29 & Good \\
\hline 9 & 45 & 26.56 & Round, 90 & 143.55 & 72.83 & 71.85 & 49.27 & 1.35 & 18 & Good \\
\hline 10 & 40 & 21.83 & Round, 90 & 110.32 & 46.68 & 45.34 & 57.69 & 2.87 & 30 & Fair \\
\hline 11 & 36 & 16.82 & Round, 90 & 86.11 & 34.64 & - & 59.77 & - & 24 & Good \\
\hline 12 & 40 & 19.14 & Anatomical, 155 & 79.69 & 39.18 & 38.37 & 50.83 & 2.07 & 25 & Good \\
\hline 13 & 43 & 23.15 & Round, 90 & 167.79 & 64.88 & - & 61.33 & - & 24 & Good \\
\hline 14 & 43 & 20.96 & Round, 90 & 119.72 & 47.56 & 42.40 & 60.27 & 10.85 & 24 & Good \\
\hline 15 & 53 & 27.85 & Anatomical, 215 & 156.71 & 71.12 & - & 54.62 & - & 19 & Good \\
\hline 16 & 51 & 26.84 & Anatomical, 155 & 112.98 & 65.67 & - & 41.87 & - & 23 & Good \\
\hline 17 & 42 & 26.50 & Anatomical, 135 & 136.05 & 69.00 & 64.00 & 49.28 & 7.25 & 18 & Good \\
\hline 18 & 51 & 19.63 & Anatomical, 190 & 99.20 & 42.60 & - & 57.06 & - & 18 & Good \\
\hline 19 & 51 & 27.06 & Anatomical, 120 & 177.31 & 93.52 & - & 47.26 & - & 21 & Good \\
\hline 20 & 52 & 27.11 & Anatomical, 190 & 134.10 & 68.85 & - & 48.66 & - & 20 & Good \\
\hline 21 & 40 & 20.55 & Round, 90 & 108.10 & 35.93 & - & 66.76 & - & 18 & Good \\
\hline 22 & 49 & 25.56 & Anatomical, 120 & 137.04 & 65.61 & - & 52.12 & - & 18 & Good \\
\hline 23 & 47 & 18.66 & Anatomical, 135 & 84.36 & 32.37 & - & 61.63 & - & 18 & Good \\
\hline Average & 44.83 & 23.01 & & 118.82 & 54.09 & 50.04 & 54.48 & 11.94 & 26.60 & \\
\hline
\end{tabular}

BMI, body mass index.

a)The decrease in volume at 6 to 8 months after surgery; biThe additional volume decrease compared to the measurements from 10 to 12 months before; ${ }^{\text {c)} C l i n i c a l ~ r e s u l t, ~}$ poor/fair/good.

\section{Fig. 1. Muscle volume measurements on axial CT}

Each image showed the transpositioned latissimus dorsi muscle, and the cross-section of the muscle was manually measured using the built-in measurement tool in the picture archiving and communications system. These values were multiplied by $5 \mathrm{~mm}$ of thickness and added together to obtain the volume. (A) Area of transpositioned latissimus dorsi muscle, immediate postoperative computed tomography (CT) view. (B) CT view of the area of the muscle 8 months after the operation. (C) Immediate postoperative view of another patient, (D) view 8 months later.
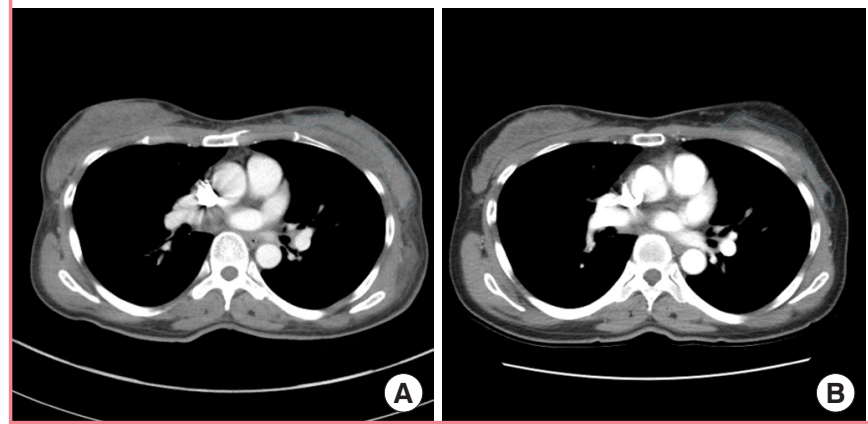

cause the contour of the implant was visible (Table 1). All the other patients were satisfied with the outcomes.

\section{DISCUSSION}

Satisfactory results using only an LDMC flap can be achieved in breast reconstructions in Asian patients with small to moderate-
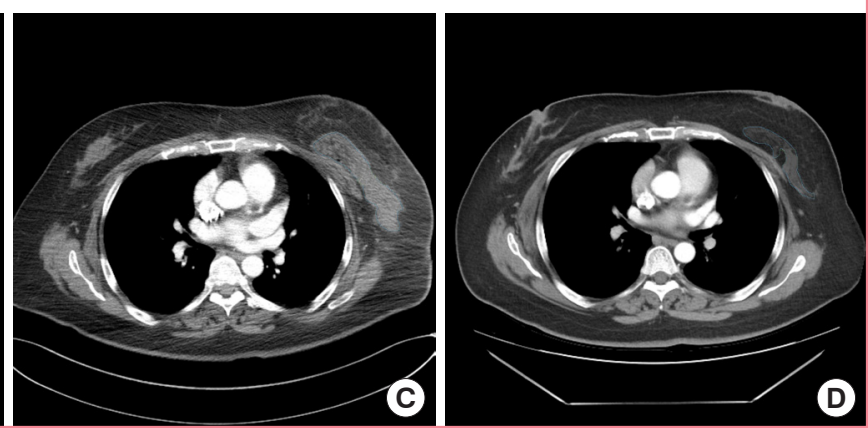

ly-sized breasts. However, for reconstructions of moderatelysized to large breasts, the additional use of implants improves the results of most procedures. The authors suggest that the results should be evaluated at least 6 to 12 months after surgery in patients who do not receive any adjuvant therapy, such as chemotherapy or radiation therapy, after breast reconstruction. When adjuvant therapy is conducted, the evaluation should be 
performed 2 years after treatment is completed. This is due to the cytotoxic effects of chemotherapy [1] and the capsular contracture, lymphedema, cellular damage, and fat necrosis resulting from radiation that can continue for a certain period of time, even after adjuvant therapy is completed. Moreover, capsular contracture and a decrease in the volume of the latissimus dorsi muscle may continue due to the use of implants, even in patients who do not receive adjuvant therapy.

The latissimus dorsi muscle is categorized as type $\mathrm{V}$ in the Mathes-Nahai classification, and the circulation provided by a single dominant pedicle is very likely to enable the flap to survive; however, due to the decrease in blood supply resulting from the ligation of multiple secondary segmental pedicles during breast reconstruction using an LDMC flap, a decrease in the muscle volume is inevitable. In order to avoid synkinetic muscle contractures in the latissimus dorsi muscle after surgery, motor nerve ligation is necessary. However, motor nerve ligation can also cause muscle atrophy due to denervation $[7,8]$. In order to overcome these effects associated with decreased muscle volume, a flap with a larger volume than that of the defect must be harvested, but objective and quantitative studies on this pattern of volume decrease are still lacking. Since most breast cancer patients undergo both breast-conserving surgery and immediate breast reconstruction along with postoperative adjuvant therapy, long-term changes in the volume of the reconstructed breast during follow-up are extremely important.

For this study, subjects were selected who received both mastectomy and immediate breast reconstruction using an LDMC flap, but did not undergo chemotherapy or radiation therapy. The extent to which the volume of the latissimus dorsi muscle decreased in the study group was observed through follow-up conducted between 6-8 months and 18 months. Through these observations, this study can serve as a useful comparative study for future research on the decrease in latissimus dorsi muscle volume in patients who undergo chemotherapy and radiation therapy. Furthermore, this study can be helpful in preoperative planning for patients who will not receive adjuvant therapy. Although the number of patients in this study may be small, since only a portion of all patients who do not have axillary lymph node metastasis and undergo mastectomy can avoid postoperative adjuvant therapy, this study is important because it can serve as a reference for future supplemental research.

Furthermore, the authors clinically observed many patients who were excluded from this study due to the lack of a single consistent CT scanning protocol. Their progress was observed at 2-year follow-ups, and upon gross examination, the majority of these patients maintained the same breast volume without changes for 6 to 18 months after surgery. The reason for this is that many patients experience weight gain due to taking tamoxifen and similar medications, and dietary and exercise habits are different for each patient. In addition, the authors restricted patients from performing exercises involving the pectoralis muscle (e.g., swimming, tennis, and upper body exercises) other than upper body stretching for at least 18 months after surgery. A wire bra should be worn for 2 years after the operation, and this postoperative management method also affects the maintenance of breast volume.

In this study, we measured the degree to which muscle volume decreased through CT scans obtained 6 to 8 months after surgery. Some patients who underwent CT scans to check for re-

Fig. 2. Follow-up after breast reconstruction

Long-term follow-up after immediate breast reconstruction with an extended latissimus dorsi musculocutaneous flap. (A) Before the breast cancer operation. (B) After 4 months. (C) After 1 year. (D) After 5 years. Upon gross examination, the majority of patients maintained the same breast volume without changes for 1 year after surgery.
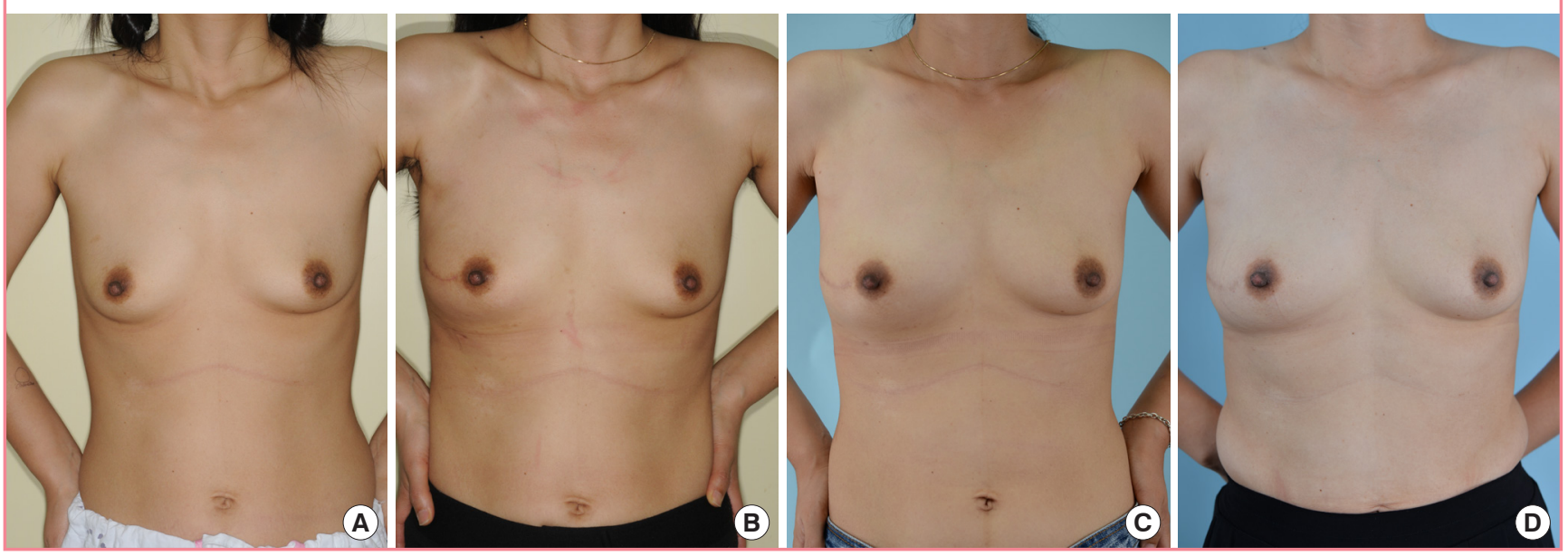
currence 24 months after surgery maintained the same breast volume, as observed in the gross examinations (Fig. 2); however, it was difficult to accurately distinguish the border of the latissimus dorsi muscle in the CT scans in most of those patients, which made it difficult to measure the volume of the pure muscle. Therefore, they were excluded from the study.

Immediate reconstruction using only an LDMC flap is possible for small to moderately-sized breasts, but most of the patients in this study used implants as well and showed favorable long-term follow-up results. The LDMC flap includes subcutaneous tissue and fat tissue, meaning that the pure muscle volume is not equivalent to the entire flap volume. However, since the detection of volume changes in the latissimus dorsi muscle is facilitated by understanding the degree of atrophy after adjuvant therapy, and since immediate breast reconstruction using both breast-conserving surgery and an LDMC flap requires chemotherapy or radiation therapy, our findings can serve as a reference for accurately assessing the volume of the latissimus dorsi muscle. This, in turn, will help improve surgical outcomes. This study observed changes in the volume of the latissimus dorsi muscle in patients who did not receive adjuvant therapy, with the goal of obtaining comparative data useful for further research.

\section{NOTES}

\section{Conflict of interest}

No potential conflict of interest relevant to this article was reported.

\section{Ethical approval}

The study was approved by the Institutional Review Board of Pusan National University Yangsan Hospital (IRB No. 05-2018051) and performed in accordance with the principles of the Declaration of Helsinki. Written informed consents were obtained.

\section{Patient consent}

The patients provided written informed consent for the publication and the use of their images.

\section{Author contribution}

Conceptualization: Nam SB. Data curation: Nam SB, Kim HY, Lee SH. Formal analysis: Oh HC. Methodology, Choi JY, Bae SH. Project administration: Bae SH. Visualization: Choo KS. Writing - original draft: Nam SB. Writing - review \& editing: Lee JW.

\section{ORCID}

Su Bong Nam https://orcid.org/0000-0002-9661-0879

Heung Chan Oh https://orcid.org/0000-0003-3209-6310

Jae Yeon Choi https://orcid.org/0000-0002-0636-998X

Seong Hwan Bae https://orcid.org/0000-0002-7203-8978

Ki Seok Choo https://orcid.org/0000-0001-5072-4259

Hyun Yul Kim https://orcid.org/0000-0001-9008-1278

Sang Hyup Lee https://orcid.org/0000-0002-9848-5469

Jae Woo Lee https://orcid.org/0000-0002-0945-6966

\section{REFERENCES}

1. Azzawi K, Ismail A, Earl H, et al. Influence of neoadjuvant chemotherapy on outcomes of immediate breast reconstruction. Plast Reconstr Surg 2010;126:1-11.

2. Mosahebi A, Ramakrishnan V, Gittos M, et al. Aesthetic outcome of different techniques of reconstruction following nipple-areola-preserving envelope mastectomy with immediate reconstruction. Plast Reconstr Surg 2007;119:796803.

3. Saulis AS, Mustoe TA, Fine NA. A retrospective analysis of patient satisfaction with immediate postmastectomy breast reconstruction: comparison of three common procedures. Plast Reconstr Surg 2007;119:1669-76.

4. Shin IS, Lee DW, Lew DH. Efficacy of quilting sutures and fibrin sealant together for prevention of seroma in extended latissimus dorsi flap donor sites. Arch Plast Surg 2012;39: 509-13.

5. Kim H, Wiraatmadja ES, Lim SY, et al. Comparison of morbidity of donor site following pedicled muscle-sparing latissimus dorsi flap versus extended latissimus dorsi flap breast reconstruction. J Plast Reconstr Aesthet Surg 2013;66:6406.

6. Yezhelyev M, Duggal CS, Carlson GW, et al. Complications of latissimus dorsi flap breast reconstruction in overweight and obese patients. Ann Plast Surg 2013;70:557-62.

7. Kaariainen M, Giordano S, Kauhanen S, et al. The significance of latissimus dorsi flap innervation in delayed breast reconstruction: a prospective randomized study-magnetic resonance imaging and histologic findings. Plast Reconstr Surg 2011;128:637e-645e.

8. Paolini G, Longo B, Laporta R, et al. Permanent latissimus dorsi muscle denervation in breast reconstruction. Ann Plast Surg 2013;71:639-42.

9. Szychta P, Butterworth M, Dixon M, et al. Breast reconstruction with the denervated latissimus dorsi musculocutaneous flap. Breast 2013;22:667-72. 